\title{
HARVESTING IN A TWO-PREY ONE-PREDATOR FISHERY: A BIOECONOMIC MODEL
}

\author{
T. K. KAR ${ }^{1}$ and K. S. CHAUDHURI ${ }^{2}$
}

(Received 22 June, 2001; revised 20 September, 2002)

\begin{abstract}
A multispecies harvesting model with interference is proposed. The model is based on Lotka-Volterra dynamics with two competing species which are affected not only by harvesting but also by the presence of a predator, the third species. In order to understand the dynamics of this complicated system, we choose to model the simplest possible predator response function in which the feeding rate of the predator increases linearly with prey density. We derive the conditions for global stability of the system using a Lyapunov function. The possibility of existence of a bioeconomic equilibrium is discussed. The optimal harvest policy is studied and the solution is derived in the equilibrium case using Pontryagin's maximal principle. Finally, some numerical examples are discussed.
\end{abstract}

\section{Introduction}

Bioeconomic modelling of the exploitation of biological resources such as fisheries and forestries has gained importance in recent years. The techniques and issues associated with the bioeconomic exploitation of these resources have been discussed in detail by Clark [5,6]. Since most marine fisheries are essentially multispecies in nature, exploitation of mixed-species fisheries has started to draw attention from researchers. Although numerous models on single species fisheries have so far appeared in the fishery literature, no fully adequate studies on multispecies fisheries appear to exist. It is very difficult to construct a realistic model of a multispecies community. Even if we succeed in formulating such a model, it is quite likely that the model may not be analytically tractable. Not every part of the catch is edible and harvesting harms some of the marine species which live on the other species in the sea. Thus the predator species are likely to become extinct with an indiscrete increase in the harvesting of prey

\footnotetext{
${ }^{1}$ Department of Mathematics, B.E. College (Deemed University), Howrah 71 1103 , India; e-mail: tkar@math.becs.ac.in.

${ }^{2}$ Department of Mathematics, Jadavpur University, Calcutta 700032, India; e-mail: jumath@cal.vsnl.net.in.

(C) Australian Mathematical Society 2004, Serial-fee code 1446-1811/04
} 
species. Therefore, how best to harvest ecologically or economically interdependent populations in the sense of maximising the present value of a stream of revenues from them, while maintaining ecological balance, is an important optimal control problem for fisheries. Clark [5] discussed an optimal equilibrium policy for the combined harvesting of two ecologically independent species. Chaudhuri $[1,2]$ formulated an optimal control problem for the combined harvesting of two competing species. Models on the combined harvesting of a two-species prey-predator fishery have been discussed by Chaudhuri and Saha Ray [4], Mesterton-Gibbons [7], Ragozin and Brown [3] etc. Most of the mathematical models on the harvesting of a multispecies fishery have so far assumed that the species are affected by harvesting only. To the authors' knowledge, no attempt has yet been made to study a harvesting model of two competing species in the presence of a predator, the third species which is not harvested.

In this paper, we study the problem of harvesting two competing species in the presence of a predator species which feeds on both the competing species. A combined harvesting effort is devoted to the exploitation of the first two (prey) species while the third (predator) species is not harvested. The problem is clearly stated in the next section. We have analysed the existence and stability of the equilibria of the system. We derive conditions for global stability of the system. Taking simple economic considerations into account, we discuss the possibilities of the existence of a bioeconomic equilibrium. The optimal policy of exploitation is derived using Pontryagin's maximal principle. Last, some numerical illustrations are given.

\section{Formulation of the problem}

The ecological system is as follows. There are two fish species which compete with each other for the use of a common resource and both of them are subjected to continuous harvesting. There is a predator (for example a whale) feeding on both of them. It is assumed that the predator population is not harvested (for example whale harvesting has been prohibited). Thus the interaction between the harvesting agency and the predator is through the third party, namely, the prey. Since we are not making a case study in respect of a specific prey-predator community, we have opted for the logistic growth function for both the prey species (that is, the population density of each prey is resource limited) and for simplicity, the feeding rate of the predator species is assumed to increase linearly with prey density.

The governing equations of the system can be written as

$$
\begin{aligned}
& d x_{1} / d t=x_{1}\left[\lambda_{1}\left(1-x_{1} / k_{1}\right)-\alpha_{12} x_{2}-\alpha_{13} x_{3}\right]-q_{1} E x_{1}, \\
& d x_{2} / d t=x_{2}\left[\lambda_{2}\left(1-x_{2} / k_{2}\right)-\alpha_{21} x_{1}-\alpha_{23} x_{3}\right]-q_{2} E x_{2}, \\
& d x_{3} / d t=x_{3}\left[\alpha_{31} x_{1}+\alpha_{32} x_{2}-x_{3}\right],
\end{aligned}
$$


where $\lambda_{1}, \lambda_{2}, k_{1}, k_{2}, \alpha_{12}, \alpha_{13}, \alpha_{21}, \alpha_{23}, \alpha_{31}$ and $\alpha_{32}$ are positive rate constants. Specifically $\lambda_{1}, \lambda_{2}$ are the biotic potentials and $k_{1}, k_{2}$ are the environmental carrying capacities of the two prey species; $\alpha_{12}, \alpha_{21}$ are the coefficients of interspecific competition between the two prey species; $\alpha_{13}, \alpha_{23}$ are the predation coefficients; $\alpha_{31}, \alpha_{32}$ are the conversion parameters; $E$ is the harvesting effort; $q_{1}, q_{2}$ are the catchability coefficients of $x_{1}$ and $x_{2}$ respectively. The catch-rate functions $q_{1} E x_{1}$ and $q_{2} E x_{2}$ are based on the CPUE (catch-per-unit-effort) hypothesis [5].

\section{The steady states}

The steady states of the system $(2.1)$ are $P_{0}(0,0,0), P_{1}\left(0, \bar{x}_{2}, \bar{x}_{3}\right), P_{2}\left(\overline{\bar{x}}_{1}, 0, \overline{\bar{x}}_{3}\right)$ and $P_{3}\left(x_{1}^{*}, x_{2}^{*}, x_{3}^{*}\right)$, where

$$
\begin{array}{lll}
\bar{x}_{2}=\frac{\lambda_{2}-q_{2} E}{\lambda_{2} / k_{2}+\alpha_{32} \alpha_{23}}, & \bar{x}_{3}=\frac{\alpha_{32}\left(\lambda_{2}-q_{2} E\right)}{\lambda_{2} / k_{2}+\alpha_{32} \alpha_{23}}, \\
\overline{\bar{x}}_{1}=\frac{\lambda_{1}-q_{1} E}{\lambda_{1} / k_{1}+\alpha_{31} \alpha_{13}}, & \overline{\bar{x}}_{3}=\frac{\alpha_{31}\left(\lambda_{1}-q_{1} E\right)}{\lambda_{1} / k_{1}+\alpha_{31} \alpha_{13}} .
\end{array}
$$

We assume here that the interior equilibrium point $\left(x_{1}^{*}, x_{2}^{*}, x_{3}^{*}\right)$ exists. There are also three other equilibria in the $x_{1} x_{2}$-plane $\left(x_{3}=0\right)$. But we are not interested in these equilibria, since for $x_{3}=0$, this is a two-species competitive model which has been studied in depth by Chaudhuri [1].

The equilibrium point $P_{1}$ exists if $E<\lambda_{2} / q_{2}$, that is, if $E<\operatorname{BTP}_{x_{2}}$ and $P_{2}$ exists if $E<\lambda_{1} / q_{1}$, that is, if $E<\operatorname{BTP}_{x_{1}}$. The ratio $(\lambda / q)$ of the biotic potential $(\lambda)$ to the catchability coefficient $(q)$ is known as the Biotechnical Productivity (BTP) of the species [5].

\section{Local stability}

The eigenvalues of the variational matrix $V(0,0,0)$ are $0, \lambda_{1}-q_{1} E$ and $\lambda_{2}-q_{2} E$ (see Appendix A). Hence the integral curves terminate in a plane corresponding to the steady state.

One of the eigenvalues of the variational matrix $V\left(0, \bar{x}_{2}, \bar{x}_{3}\right)$ is $\lambda_{1}-\alpha_{12} \bar{x}_{2}-\alpha_{13} \bar{x}_{3}-$ $q_{1} E$ (see Appendix $\mathrm{B}$ ). This eigenvalue is negative or positive according to whether $\lambda_{1} / q_{1}$ is less or greater than $\left(\alpha_{12} \bar{x}_{2}+\alpha_{13} \bar{x}_{3}\right) / q_{1}+E$.

The other two eigenvalues are given by the roots of the following quadratic equation

$$
\mu^{2}+\mu\left(\lambda_{2} \bar{x}_{2} / k_{2}+\bar{x}_{3}\right)+\left(\lambda_{2} / k_{2}+\alpha_{23} \alpha_{32}\right) \bar{x}_{2} \cdot \bar{x}_{3}=0 .
$$

In (4.1), the sum of the roots $=-\left(\lambda_{2} \bar{x}_{2} / k_{2}+\bar{x}_{3}\right)$, which is always negative and the product of the roots $=\left(\lambda_{2} / k_{2}+\alpha_{23} \alpha_{32}\right) \bar{x}_{2} \bar{x}_{3}$, which is always positive. 
Therefore the roots of (4.1) are real and negative or complex conjugates having negative real parts. Thus $P_{1}$ is asymptotically stable only if

$$
\lambda_{1} / q_{1}<\left(\alpha_{12} \bar{x}_{2}+\alpha_{13} \bar{x}_{3}\right) / q_{1}+E \text {. }
$$

We have already found that the steady state $P_{1}$ exists if $E<\lambda_{2} / q_{2}$. Hence the condition for asymptotic stability of $P_{1}$ becomes

$$
\frac{\lambda_{1}}{q_{1}}-\frac{\alpha_{12} \bar{x}_{2}+\alpha_{13} \bar{x}_{3}}{q_{1}}<E<\frac{\lambda_{2}}{q_{2}} .
$$

This defines a range in which the fishing effort must lie to ensure a stable equilibrium $P_{1}$.

One of the eigenvalues of the variational matrix $V\left(\overline{\bar{x}}_{1}, 0, \overline{\bar{x}}_{3}\right)$ is $\lambda_{2}-\alpha_{21} \overline{\bar{x}}_{1}-\alpha_{23} \overline{\bar{x}}_{3}-$ $q_{2} E$ (see Appendix $C$ ). This eigenvalue is negative or positive according to whether $\lambda_{2} / q_{2}$ is less or greater than $\left(\alpha_{21} \overline{\bar{x}}_{1}+\alpha_{23} \overline{\bar{x}}_{3}\right) / q_{2}+E$. The other two eigenvalues are given by the roots of the quadratic equation

$$
\mu^{2}+\left(\lambda_{1} \overline{\bar{x}}_{1} / k_{1}+\overline{\bar{x}}_{3}\right) \mu+\left(\lambda_{1} / k_{1}+\alpha_{31} \alpha_{13}\right) \overline{\bar{x}}_{1} \overline{\bar{x}}_{3}=0
$$

In (4.2), the sum of the roots $=-\left(\lambda_{1} \overline{\bar{x}}_{1} / k_{1}+\overline{\bar{x}}_{3}\right)$, which is always negative and the product of the roots $=\left(\lambda_{1} / k_{1}+\alpha_{31} \alpha_{13}\right) \overline{\bar{x}}_{1} \overline{\bar{x}}_{3}$, which is always positive.

Therefore the roots of (4.2) are real and negative or complex conjugates having negative real parts. Thus $P_{2}$ is asymptotically stable only if

$$
\lambda_{2} / q_{2}<\left(\alpha_{21} \overline{\bar{x}}_{1}+\alpha_{23} \overline{\bar{x}}_{3}\right) / q_{2}+E \text {. }
$$

Since $P_{2}$ exists only if $E<\lambda_{1} / q_{1}$, the condition for asymptotic stability of $P_{2}$ becomes

$$
\lambda_{2} / q_{2}-\left(\alpha_{21} \overline{\bar{x}}_{1}+\alpha_{23} \overline{\bar{x}}_{3}\right) / q_{2}<E<\lambda_{1} / q_{1} .
$$

The effort level must lie within this range for the existence of a stable steady state $P_{2}$. The characteristic equation for the variational matrix $V\left(x_{1}^{*}, x_{2}^{*}, x_{3}^{*}\right)$ is $b_{3} \mu^{3}+$ $b_{2} \mu^{2}+b_{1} \mu+b_{0}=0$ (see Appendix D).

Using the Routh-Hurwitz criteria [8], it can be shown that $P_{3}$ is stable if

(i) $\lambda_{1} / k_{1}>\alpha_{12} \alpha_{31} / \alpha_{32}$ and

(ii) $\lambda_{2} / k_{2}>\alpha_{21} \alpha_{32} / \alpha_{31}$.

\section{Global stability}

In this section, we shall prove the global stability of the system (2.1) by constructing a suitable Lyapunov function. 
THEOREM 1. The interior equilibrium point $P_{3}$ is globally asymptotically stable if

(i) $\alpha_{13}=\alpha_{31}, \alpha_{23}=\alpha_{32}$ and

(ii) $\left(4 \lambda_{1} \lambda_{2}\right) /\left(k_{1} k_{2}\right)>\left(\alpha_{12}+\alpha_{21}\right)^{2}$.

PrOOF. Let us consider a suitable Lyapunov function

$$
\begin{aligned}
v\left(x_{1}, x_{2}, x_{3}\right)= & \left(x_{1}-x_{1}^{*}\right)-x_{1}^{*} \log \left(x_{1} / x_{1}^{*}\right)+\left(x_{2}-x_{2}^{*}\right)-x_{2}^{*} \log \left(x_{2} / x_{2}^{*}\right) \\
& +\left(x_{3}-x_{3}^{*}\right)-x_{3}^{*} \log \left(x_{3} / x_{3}^{*}\right)
\end{aligned}
$$

Obviously $v$ is positive definite.

The time derivative of $v$ along the solutions of (2.1), after a little simplification, is given by

$$
\begin{aligned}
\frac{d v}{d t}=- & {\left[\lambda_{1}\left(x_{1}-x_{1}^{*}\right)^{2} / k_{1}+\left(x_{1}-x_{1}^{*}\right)\left(x_{2}-x_{2}^{*}\right)\left(\alpha_{12}+\alpha_{21}\right)\right.} \\
& +\lambda_{2}\left(x_{2}-x_{2}^{*}\right)^{2} / k_{2}+\left(x_{2}-x_{2}^{*}\right)\left(x_{3}-x_{3}^{*}\right)\left(\alpha_{23}-\alpha_{32}\right) \\
& \left.+\left(x_{3}-x_{3}^{*}\right)^{2}+\left(x_{3}-x_{3}^{*}\right)\left(x_{1}-x_{1}^{*}\right)\left(\alpha_{13}-\alpha_{31}\right)\right]
\end{aligned}
$$

The right-hand side of (5.1) can be written as $-X^{T} A X$, where

$$
X^{T}=\left[\left(x_{1}-x_{1}^{*}\right),\left(x_{2}-x_{2}^{*}\right),\left(x_{3}-x_{3}^{*}\right)\right]
$$

and

$$
A=\left[\begin{array}{ccc}
\lambda_{1} / k_{1} & \left(\alpha_{12}+\alpha_{21}\right) / 2 & \left(\alpha_{13}-\alpha_{31}\right) / 2 \\
\left(\alpha_{12}+\alpha_{21}\right) / 2 & \lambda_{2} / k_{2} & \left(\alpha_{23}-\alpha_{32}\right) / 2 \\
\left(\alpha_{13}-\alpha_{31}\right) / 2 & \left(\alpha_{23}-\alpha_{32}\right) / 2 & 1
\end{array}\right]
$$

Therefore $d v / d t<0$ if $A$ is positive definite. The matrix $A$ is positive definite if the hypotheses of Theorem 1 are satisfied.

\section{Bionomic equilibrium}

The term bionomic equilibrium is an amalgamation of the concepts of biological equilibrium as well as economic equilibrium. As we already saw, a biological equilibrium is given by $\dot{x}_{1}=0, \dot{x}_{2}=0, \dot{x}_{3}=0$. The economic equilibrium is said to be achieved when TR (the total revenue obtained by selling the harvested biomass) equals TC (the total cost for the effort devoted to harvesting).

Let $C=$ constant fishing cost per unit effort, $p_{1}=$ constant price per unit biomass of the first species, and $p_{2}=$ constant price per unit biomass of the second species. 
The economic rent (net revenue) at any time is given by

$$
\pi\left(x_{1}, x_{2}, x_{3}, E\right)=\mathrm{TR}-\mathrm{TC}=\left(p_{1} q_{1} x_{1}+p_{2} q_{2} x_{2}-C\right) E .
$$

Now,

$$
\begin{aligned}
& \dot{x}_{1}=0 \Rightarrow x_{1}=0 \quad \text { or } \quad E=\frac{\lambda_{1}}{q_{1}}-\frac{\lambda_{1}}{k_{1} q_{1}} x_{1}-\frac{\alpha_{12}}{q_{1}} x_{2}-\frac{\alpha_{13}}{q_{1}} x_{3} \text {, } \\
& \dot{x}_{2}=0 \Rightarrow x_{2}=0 \quad \text { or } \quad E=\frac{\lambda_{2}}{q_{2}}-\frac{\lambda_{2}}{k_{2} q_{2}} x_{2}-\frac{\alpha_{21}}{q_{2}} x_{1}-\frac{\alpha_{23}}{q_{2}} x_{3} \text {, } \\
& \dot{x}_{3}=0 \Rightarrow x_{3}=0 \text { or } \quad x_{3}=\alpha_{31} x_{1}+\alpha_{32} x_{2} \text {. }
\end{aligned}
$$

Hence the nontrivial biological equilibrium solution occurs at a point on the line

$$
\left.\begin{array}{r}
\left(\lambda_{1} / k_{1} q_{1}-\alpha_{21} / q_{2}\right) x_{1}-\left(\lambda_{2} / q_{2}-\alpha_{12} / q_{1}\right) x_{2} \\
+\left(\alpha_{13} / q_{1}-\alpha_{23} / q_{2}\right) x_{3}+\left(\lambda_{2} / q_{2}-\lambda_{1} / q_{1}\right)=0, \\
\alpha_{31} x_{1}+\alpha_{32} x_{2}-x_{3}=0,
\end{array}\right\}
$$

where $0 \leq x_{1} \leq k_{1}, 0 \leq x_{2} \leq k_{2}$.

The equilibrium line (6.1) meets the plane $x_{1}=0$ at $\left(0, \tilde{x}_{2}, \tilde{x}_{3}\right)$, where

$$
\tilde{x}_{2}=\frac{\lambda_{2} / q_{2}-\lambda_{1} / q_{1}}{\left(\lambda_{2} / k_{2} q_{2}+\alpha_{23} \alpha_{32} / q_{2}\right)-\left(\alpha_{12} / q_{1}+\alpha_{31} \alpha_{32} / q_{1}\right)}, \quad \tilde{x}_{3}=\alpha_{32} \tilde{x}_{2}
$$

provided either

(a) $\lambda_{2} / q_{2}>\max \left(\lambda_{1} / q_{1}, k_{2} \alpha_{12} / q_{1}\right)$ and $\alpha_{23} / q_{2}>\alpha_{13} / q_{1}$ or

(b) $\lambda_{2} / q_{2}<\min \left(\lambda_{1} / q_{1}, k_{2} \alpha_{12} / q_{1}\right)$ and $\alpha_{23} / q_{2}<\alpha_{13} / q_{1}$ hold.

Similarly, (6.1) meets the plane $x_{2}=0$ at $\left(\tilde{x}_{1}, 0, \tilde{x}_{3}\right)$, where

$$
\tilde{x}_{1}=\frac{\lambda_{2} / q_{2}-\lambda_{1} / q_{1}}{\left(\alpha_{21} / q_{2}+\alpha_{23} \alpha_{31} / q_{2}\right)-\left(\lambda_{1} / k_{1} q_{1}+\alpha_{13} \alpha_{31} / q_{1}\right)}, \quad \tilde{x}_{3}=\alpha_{31} \tilde{x}_{1}
$$

provided either

(c) $\lambda_{1} / q_{1}>\max \left(\lambda_{2} / q_{2}, k_{1} \alpha_{21} / q_{2}\right)$ and $\alpha_{13} / q_{1}>\alpha_{23} / q_{2}$ or

(d) $\lambda_{1} / q_{1}<\min \left(\lambda_{2} / q_{2}, k_{1} \alpha_{21} / q_{2}\right)$ and $\alpha_{13} / q_{1}<\alpha_{23} / q_{2}$ hold.

The bionomic equilibrium $R\left(x_{1 \infty}, x_{2 \infty}, x_{3 \infty}\right)$ will be the point of intersection (if it exists) of (6.1) and $\pi\left(x_{1}, x_{2}, x_{3}, E\right)=\left(p_{1} q_{1} x_{1}+p_{2} q_{2} x_{2}-C\right) E=0$ in the first octant.

After a little calculation, it is seen that $x_{1 \infty}>0$ provided either

(e) $\left(\frac{\lambda_{2}}{k_{2} q_{2}}+\frac{\alpha_{32} \alpha_{23}}{q_{2}}-\frac{\alpha_{12}}{q_{1}}-\frac{\alpha_{13} \alpha_{32}}{q_{1}}\right)$

or

$$
>\max \left[\frac{p_{2} q_{2}}{p_{1} q_{1}}\left(\frac{\alpha_{21}}{q_{1}}+\frac{\alpha_{23} \alpha_{31}}{q_{2}}-\frac{\lambda_{1}}{k_{1} q_{1}}-\frac{\alpha_{13} \alpha_{31}}{q_{1}}\right), \frac{p_{2} q_{2}}{c}\left(\frac{\lambda_{2}}{q_{2}}-\frac{\lambda_{1}}{q_{1}}\right)\right]
$$


(f) $\left(\frac{\lambda_{2}}{k_{2} q_{2}}+\frac{\alpha_{32} \alpha_{23}}{q_{2}}-\frac{\alpha_{12}}{q_{1}}-\frac{\alpha_{13} \alpha_{32}}{q_{1}}\right)$

holds.

$$
<\min \left[\frac{p_{2} q_{2}}{p_{1} q_{1}}\left(\frac{\alpha_{21}}{q_{2}}+\frac{\alpha_{23} \alpha_{31}}{q_{2}}-\frac{\lambda_{1}}{k_{1} q_{1}}-\frac{\alpha_{13} \alpha_{31}}{q_{1}}\right), \frac{p_{2} q_{2}}{c}\left(\frac{\lambda_{2}}{q_{2}}-\frac{\lambda_{1}}{q_{1}}\right)\right]
$$

When either (a) or (b) holds but neither (e) nor (f) holds, the $x_{1}$-species faces extinction in the bionomic equilibrium. Proceeding in a similar manner, one can easily prove the possibility of extinction of the $x_{2}$-species.

Thus the combined harvesting of two competing fish species in the presence of a predator may drive one species to extinction while the bionomic equilibrium of the open-access fishing continues with the support of the other species. This phenomenon has been noticed by Clark [5] also in the case of combined harvesting of two ecologically independent species. Explicit biological or bioeconomic interpretations of the conditions (a)-(f) seems to be difficult. These may simply be regarded as some conditions to be satisfied by the biological, technical and economic parameters for the existence of a bionomic equilibrium.

\section{Optimal harvesting policy}

The present value $J$ of a continuous time-stream of revenues is given by

$$
J=\int_{0}^{\infty} e^{-\delta t}\left[p_{1} q_{1} x_{1}+p_{2} q_{2} x_{2}-C\right] E d t
$$

where $\delta$ denotes the instantaneous annual rate of discount [10]. Our problem is to maximise $J$ subject to the state equations (2.1) by invoking Pontryagin's maximal principle [9]. The control variable $E(t)$ is subjected to the constraints $0 \leq E(t) \leq$ $E_{\max }$, so that $V_{t}=\left[0, E_{\max }\right]$ is the control set.

The Hamiltonian for the problem is given by

$$
\begin{aligned}
H= & e^{-\delta t}\left[p_{1} q_{1} x_{1}+p_{2} q_{2} x_{2}-C\right] E \\
& +\mu_{1}\left(-\lambda_{1} x_{1}^{2} / k_{1}-\alpha_{12} x_{1} x_{2}-\alpha_{13} x_{1} x_{3}+x_{1}\left(\lambda_{1}-q_{1} E\right)\right) \\
& +\mu_{2}\left(-\lambda_{2} x_{2}^{2} / k_{2}-\alpha_{21} x_{1} x_{2}-\alpha_{23} x_{2} x_{3}+x_{2}\left(\lambda_{2}-q_{2} E\right)\right) \\
& +\mu_{3}\left(-x_{3}^{2}-\alpha_{31} x_{1} x_{3}-\alpha_{32} x_{2} x_{3}\right),
\end{aligned}
$$

where $\mu_{i}(t), i=1,2,3$, are the adjoint variables.

The adjoint equations are

$$
\begin{aligned}
\frac{d \mu_{1}}{d t}= & -\frac{\partial H}{\partial x_{1}} \\
= & -\left[e^{-\delta t} p_{1} q_{1} E-\mu_{1}\left(2 \lambda_{1} x_{1} / k_{1}+\alpha_{12} x_{2}+\alpha_{13} x_{3}-\left(\lambda_{1}-q_{1} E\right)\right)\right. \\
& \left.-\mu_{2} \alpha_{21} x_{2}+\mu_{3} \alpha_{31} x_{3}\right],
\end{aligned}
$$


450

$$
\begin{aligned}
\frac{d \mu_{2}}{d t}= & -\frac{\partial H}{\partial x_{2}} \\
= & -\left[e^{-\delta t} p_{2} q_{2} E-\mu_{2}\left(2 \lambda_{2} x_{2} / k_{2}+\alpha_{21} x_{1}+\alpha_{23} x_{3}-\left(\lambda_{2}-q_{2} E\right)\right)\right. \\
& \left.-\mu_{1} \alpha_{12} x_{1}+\mu_{3} \alpha_{32} x_{3}\right],
\end{aligned}
$$

and

$$
\begin{aligned}
\frac{d \mu_{3}}{d t} & =-\frac{\partial H}{\partial x_{3}} \\
& =-\left[-\mu_{1} \alpha_{13} x_{1}-\mu_{2} \alpha_{23} x_{2}-\mu_{3}\left(2 x_{3}-\alpha_{31} x_{1}-\alpha_{32} x_{2}\right)\right] .
\end{aligned}
$$

Here we deal with an optimal equilibrium solution. Since we are considering an equilibrium solution, $x_{1}, x_{2}$ and $x_{3}$ are to be treated as constants in the subsequent steps.

Now, by eliminating $\mu_{1}$ and $\mu_{2}$ from (7.2)-(7.4), we get a reduced differential equation for $\mu_{3}$ as

$$
\left(a_{3} D^{3}+3 a_{2} D^{2}+3 a_{1} D+a_{0}\right) \mu_{3}=M_{3} e^{-\delta t},
$$

where $D \equiv d / d t, a_{3}=1,3 a_{2}=-\left(x_{3}+\lambda_{2} x_{2} / k_{2}+\lambda_{1} x_{1} / k_{1}\right)$,

$$
\begin{gathered}
3 a_{1}=\frac{\lambda_{1} \lambda_{2}}{k_{1} k_{2}} x_{1} x_{2}+\frac{\lambda_{2}}{k_{2}} x_{2} x_{3}+\frac{\lambda_{1}}{k_{1}} x_{1} x_{3}-\alpha_{21} \alpha_{12} x_{1} x_{2}+\alpha_{13} \alpha_{31} x_{1} x_{3}+\alpha_{32} \alpha_{23} x_{2} x_{3} \\
a_{0}=-\left(\frac{\lambda_{2} \lambda_{1}}{k_{2} k_{1}}-\alpha_{12} \alpha_{21}+\frac{\lambda_{2}}{k_{2}} \alpha_{13} \alpha_{31}+\frac{\lambda_{1}}{k_{1}} \alpha_{32} \alpha_{23}-\alpha_{32} \alpha_{21} \alpha_{13}-\alpha_{31} \alpha_{13} \alpha_{12}\right) x_{1} x_{2} x_{3} \\
M_{3}=p_{1} q_{1}\left[x_{2} \alpha_{23} \delta+\left(-\alpha_{13} \alpha_{21}+\lambda_{1} \alpha_{23} / k_{1}\right) x_{1} x_{2}\right] E \\
+p_{2} q_{2}\left[\alpha_{13} x_{1} \delta+\left(-\alpha_{23} \alpha_{12}+\lambda_{2} \alpha_{13} / k_{2}\right) x_{1} x_{2}\right] E
\end{gathered}
$$

The complete solution of (7.5) is

$$
\mu_{3}=A_{1} e^{m_{1} t}+A_{2} e^{m_{2} t}+A_{3} e^{m_{3} t}+\left(M_{3} / N\right) e^{-\delta t},
$$

where $A_{i}(i=1,2,3)$ are arbitrary constants and $m_{i}(i=1,2,3)$ are the roots of the auxiliary equations $a_{3} m^{3}+3 a_{2} m^{2}+3 a_{1} m+a_{0}=0$ and $N=\delta^{3}-3 a_{2} \delta^{2}-3 a_{1} \delta-a_{0} \neq 0$.

It is clear from (7.6) that $\mu_{3}$ is bounded if and only if $m_{i}<0,(i=1,2,3)$ or the $A_{i}$ 's are identically equal to zero. It being very difficult to check whether $m_{i}<0$, we take $A_{i}=0(i=1,2,3)$. Then $\mu_{3}=M_{3} e^{-\delta t} / N$.

By a similar process, we get $\mu_{2}=M_{2} e^{-\delta t} / N$ and $\mu_{1}=M_{1} e^{-\delta t} / N$, where

$$
\begin{aligned}
M_{2}= & p_{1} q_{1}\left[\alpha_{12} x_{1} \delta+\left(\alpha_{12}+\alpha_{32} \alpha_{13}\right) x_{1} x_{3}\right] E \\
& -p_{2} q_{2}\left[\delta^{2}+\left(x_{3}+\lambda_{1} x_{1} / k_{1}\right) \delta+\left(\lambda_{1} / k_{1}+\alpha_{13} \alpha_{31}\right) x_{1} x_{3}\right] E
\end{aligned}
$$

and

$$
\begin{aligned}
M_{1}= & p_{2} q_{2}\left[\alpha_{21} x_{2} \delta+\left(\alpha_{21}+\alpha_{31} \alpha_{23}\right) x_{3} x_{2}\right] E \\
& -p_{1} q_{1}\left[\delta^{2}+\left(x_{3}+\lambda_{2} x_{2} / k_{2}\right) \delta+\left(\lambda_{2} / k_{2}+\alpha_{32} \alpha_{23}\right) x_{2} x_{3}\right] E .
\end{aligned}
$$


We find the shadow prices $\mu_{i}(t) e^{\delta t}, i=1,2,3$, of the three fish species remain bounded as $t \rightarrow \infty$ and hence satisfy the transversality condition at $\infty$ [5].

The Hamiltonian in (7.1) must be maximised for $E \in\left[0, E_{\max }\right]$. Assuming that the control constraints $0 \leq E \leq E_{\max }$ are not binding (that is, the optimal equilibrium does not occur either at $E=0$ or $E=E_{\max }$ ), we have singular control given by [5]. Therefore

$$
\frac{\partial H}{\partial E}=e^{-\delta t}\left(p_{1} q_{1} x_{1}+p_{2} q_{2} x_{2}-C\right)-\mu_{1} q_{1} x_{1}-\mu_{2} q_{2} x_{2}=0
$$

or $e^{-\delta t}(d \pi / d E)=\mu_{1} q_{1} x_{1}+\mu_{2} q_{2} x_{2}$.

This indicates that the total user cost of harvest per unit effort must be equal to the discounted value of the future profit at the steady-state effort level [5].

Substituting $\mu_{1}$ and $\mu_{2}$ into (7.7) we get

$$
x_{1} q_{1}\left(p_{1}-M_{1} / N\right)+x_{2} q_{2}\left(p_{2}-M_{2} / N\right)=C .
$$

The value of $E$ at the interior equilibrium given by

$$
\begin{aligned}
E & =\left[\lambda_{1}\left(1-x_{1} / k_{1}\right)-\alpha_{12} x_{2}-\alpha_{13} x_{3}\right] / q_{1} \\
& =\left[\lambda_{2}\left(1-x_{2} / k_{2}\right)-\alpha_{21} x_{1}-\alpha_{23} x_{3}\right] / q_{2}
\end{aligned}
$$

is to be substituted in the expressions for $M_{1}$ and $M_{2}$. We may then solve (7.8) and (6.1) to obtain the optimal equilibrium solution $x_{1}=x_{1 \delta}, x_{2}=x_{2 \delta}, x_{3}=x_{3 \delta}$ for a given value of $\delta$. For different values of $\delta$ we have different optimal equilibria. When $\delta \rightarrow \infty,(7.8)$ leads to the obvious result $p_{1} q_{1} x_{1 \infty}+p_{2} q_{2} x_{2 \infty}=C$ that implies $\pi\left(x_{1 \infty}, x_{2 \infty}, x_{2 \infty}, E\right)=0$.

This shows that an infinite discount rate leads to complete dissipation of economic revenue. This conclusion was also drawn by Clark [5] in the combined harvesting of two ecologically independent populations and by Chaudhuri [1] in the combined harvesting of two competing species.

Using (7.8), we have

$$
\pi=\left(p_{1} q_{1} x_{1}+p_{2} q_{2} x_{2}-C\right) E=\frac{\left(M_{1} q_{1} x_{1}+M_{2} q_{2} x_{2}\right) E}{N} .
$$

Here we note that each of $M_{1}$ and $M_{2}$ is $0\left(\delta^{2}\right)$ where $N$ is $0\left(\delta^{3}\right)$ so that $\pi$ is $0\left(\delta^{-1}\right)$. Thus $\pi$ is a decreasing function of $\delta(\geq 0)$. We therefore conclude that $\delta=0$ leads to maximisation of $\pi$.

\section{Numerical examples}

Let $\lambda_{1}=2.09, \lambda_{2}=2.07, k_{1}=200, k_{2}=300, q_{1}=0.04, q_{2}=0.01, \alpha_{12}=0.001$, $\alpha_{21}=0.001, \alpha_{13}=0.01, \alpha_{31}=0.30, \alpha_{23}=0.02, \alpha_{32}=0.3, p_{1}=6, p_{2}=8, E=10$, $C=50$ and $\delta=0.05$. 


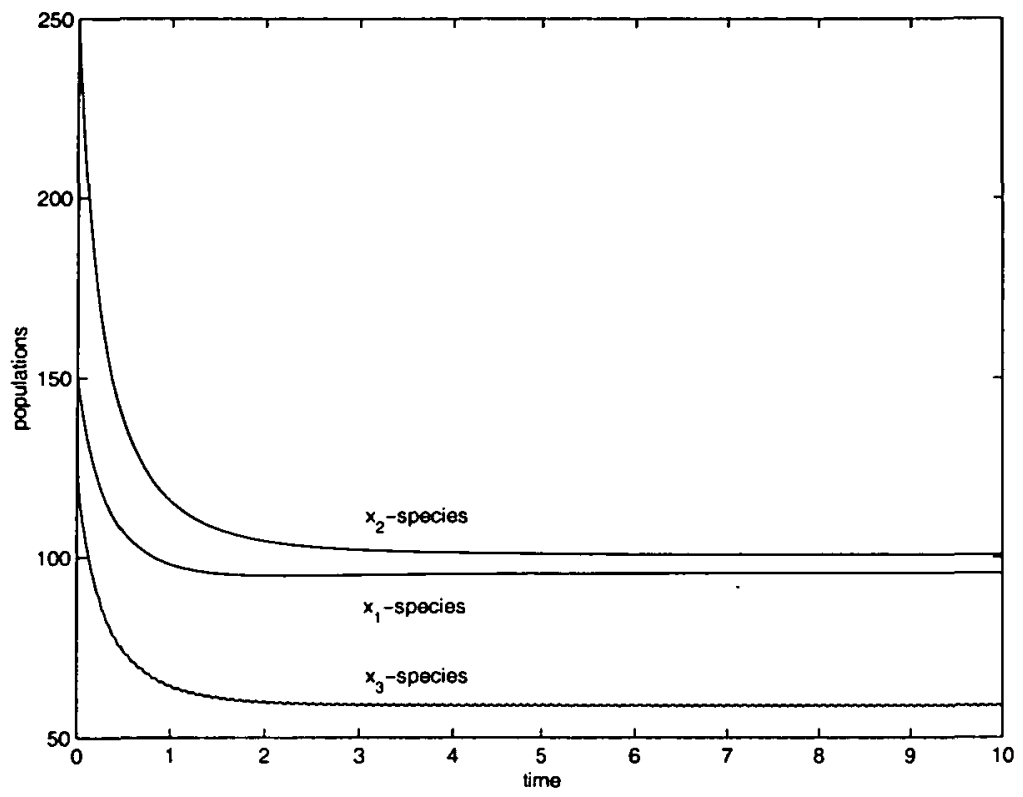

FIGURE 1. Variation of the populations against time, beginning with $x_{1}=150, x_{2}=250$ and $x_{3}=130$. Parameter values are the same as in Example 1.

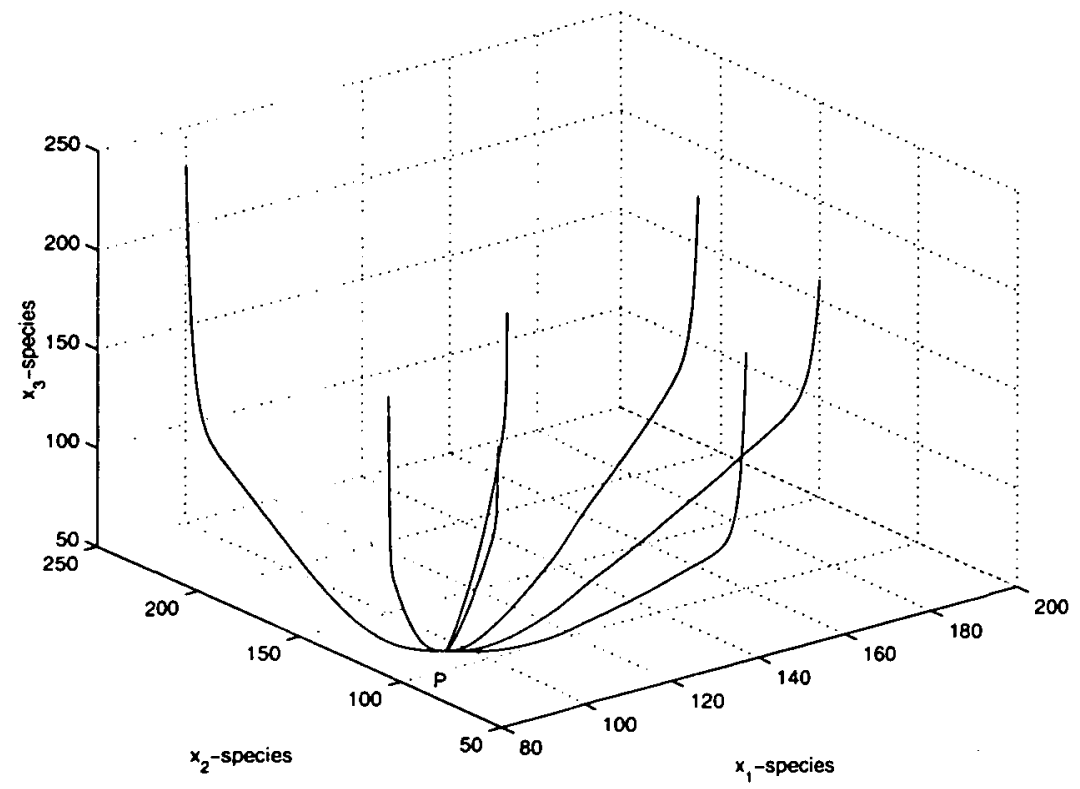

FIGURE 2. Phase-space trajectorics corresponding to the optimal harvesting effort $E=11.43$ units, with reference to different initial levels. The trajectories clearly indicate that the optimal equilibrium $P(91,102,58)$ is asymptotically stable. 
EXAMPLE 1. For the above parameter values, it is found that

(i) $P_{0}(0,0,0)$ is unstable,

(ii) $P_{1}(0,153,46)$ is unstable,

(iii) $P_{2}(162,0,48)$ is unstable,

but (iv) the only interior steady state $P_{3}(96,101,59)$ is stable.

EXAMPLE 2. For the same parameter values as those used in Example 1 we find that the bionomic equilibrium $R(184,74,77)$ and the optimal equilibrium $(91,102,58)$ both exist. We also find that the optimal harvesting effort, $E$, which leads the system to the optimal equilibrium $(91,102,58)$, is 11.43 units.

\section{Concluding remarks}

In this paper, we have attempted to study the effects of harvesting in a two-species competitive system in the presence of a predator species. We have first studied the existence and stability (local as well as global) of the possible steady states.

We then examined the possibilities of the existence of a bionomic (biological as well as economic) equilibrium of the exploited system.

Next, the optimal harvest policy was discussed. The present value of a continuous time-stream of revenues is maximised by invoking Pontryagin's maximum principle. The case of an optimal equilibrium solution is studied. It is found that the shadow prices remain constant over time in optimal equilibrium when they satisfy the transversality condition. Also the total user cost of harvest per unit effort equals the discounted value of the future profit at the steady state effort level. It is proved that zero discounting leads to maximisation of economic revenue and that an infinite discount rate leads to complete dissipation of economic rent.

Last, some numerical examples are taken to obtain steady states, bionomic equilibrium, optimal equilibrium etc. To get the numerical results, we used Lingo and Matlab.

We have established the existence of an equilibrium solution that satisfies the necessary conditions of the maximum principle. As pointed out by Clark [5], it is extremely difficult to find an optimal approach path consisting of a combination of bang-bang controls and non-equilibrium singular controls. It is also difficult to carry out dynamic optimisation [2] taking the effort level $E$ to be a dynamic (that is, time-dependent) variable. Due to these difficulties we have considered the optimal equilibrium solution only. The model can also be improved by assuming that the fishing effort $E$ increases or decreases in proportion to the flow of net economic revenue from the fishery. For such a dynamic reaction model $([3,5])$, one has to introduce a fourth differential equation in (2.1) for $d E / d t$. It is quite likely that such 
a complicated system may prove to be too formidable to solve.

\section{Appendix A.}

The variational matrix of the system of equations (2.1) is

$$
V\left(x_{1}, x_{2}, x_{3}\right)=\left[\begin{array}{ccc}
V_{11} & -\alpha_{12} x_{1} & -\alpha_{13} x_{3} \\
-\alpha_{21} x_{2} & V_{22} & -\alpha_{23} x_{2} \\
\alpha_{31} x_{3} & \alpha_{32} x_{3} & \left(\alpha_{31} x_{1}+\alpha_{32} x_{2}-2 x_{3}\right)
\end{array}\right] \text {, }
$$

where

$$
\begin{aligned}
& V_{11}=\left(\lambda_{1}-\frac{2 \lambda_{1}}{k_{1}} x_{1}-\alpha_{12} x_{2}-\alpha_{13} x_{3}-q_{1} E\right), \\
& V_{22}=\left(\lambda_{2}-\frac{2 \lambda_{2}}{k_{2}} x_{2}-\alpha_{21} x_{1}-\alpha_{23} x_{3}-q_{2} E\right) .
\end{aligned}
$$

Therefore

$$
V(0,0,0)=\left[\begin{array}{ccc}
\lambda_{1}-q_{1} E & 0 & 0 \\
0 & \lambda_{2}-q_{2} E & 0 \\
0 & 0 & 0
\end{array}\right]
$$

The eigenvalues of this variational matrix are $0, \lambda_{1}-q_{1} E$ and $\lambda_{2}-q_{2} E$.

\section{Appendix B.}

From (A.1), we have

$$
V\left(0, \bar{x}_{2}, \bar{x}_{3}\right)=\left[\begin{array}{ccc}
\lambda_{1}-\alpha_{12} \bar{x}_{2}-\alpha_{13} \bar{x}_{3}-q_{1} E & 0 & 0 \\
-\alpha_{21} \bar{x}_{2} & -\lambda_{2} \bar{x}_{2} / k_{2} & -\alpha_{23} \bar{x}_{2} \\
\alpha_{31} \bar{x}_{3} & \alpha_{32} \bar{x}_{3} & -\bar{x}_{3}
\end{array}\right] .
$$

The characteristic equation is

$$
\begin{aligned}
& {\left[\left(\lambda_{1}-\alpha_{12} \bar{x}_{2}-\alpha_{13} \bar{x}_{3}-q_{1} E\right)-\mu\right]} \\
& \quad \times\left[\mu^{2}+\mu\left(\frac{\lambda_{2}}{k_{2}} \bar{x}_{2}+\bar{x}_{3}\right)+\left(\frac{\lambda_{2}}{k_{2}} \bar{x}_{2} \bar{x}_{3}+\alpha_{23} \alpha_{32} \bar{x}_{2} \bar{x}_{3}\right)\right]=0 .
\end{aligned}
$$

Appendix C.

From (A.1), we have

$$
V\left(\overline{\bar{x}}_{1}, 0, \overline{\bar{x}}_{3}\right)=\left[\begin{array}{ccc}
-\lambda_{1} \overline{\bar{x}}_{1} / k_{1} & -\alpha_{12} \overline{\bar{x}}_{1} & -\alpha_{13} \overline{\bar{x}}_{1} \\
0 & \lambda_{2}-\alpha_{21} \overline{\bar{x}}_{1}-\alpha_{23} \overline{\bar{x}}_{3}-q_{2} E & 0 \\
\alpha_{31} \overline{\bar{x}}_{3} & \alpha_{32} \overline{\bar{x}}_{3} & -\overline{\bar{x}}_{3}
\end{array}\right] .
$$


The characteristic equation is

$$
\begin{aligned}
& {\left[\lambda_{2}-\alpha_{21} \overline{\bar{x}}_{1}-\alpha_{23} \overline{\bar{x}}_{3}-q_{2} E-\mu\right]} \\
& \quad \times\left[\mu^{2}+\mu\left(\frac{\lambda_{1}}{k_{1}} \overline{\bar{x}}_{1}+\overline{\bar{x}}_{3}\right)+\left(\frac{\lambda_{1}}{k_{1}} \overline{\bar{x}}_{1} \overline{\bar{x}}_{3}+\alpha_{31} \alpha_{13} \overline{\bar{x}}_{1} \overline{\bar{x}}_{3}\right)\right]=0 .
\end{aligned}
$$

\section{Appendix D.}

From (A.1), we have

$$
V\left(x_{1}^{*}, x_{2}^{*}, x_{3}^{*}\right)=\left[\begin{array}{ccc}
-\lambda_{1} x_{1}^{*} / k_{1} & -\alpha_{12} x_{1}^{*} & -\alpha_{13} x_{1}^{*} \\
-\alpha_{21} x_{2}^{*} & -\lambda_{2} x_{2}^{*} / k_{2} & -\alpha_{23} x_{2}^{*} \\
\alpha_{31} x_{3}^{*} & \alpha_{32} x_{3}^{*} & -x_{3}^{*}
\end{array}\right] .
$$

The characteristic equation is

$$
\begin{aligned}
& \mu^{3}+\mu^{2}\left(\frac{\lambda_{2}}{k_{2}} x_{2}^{*}+\frac{\lambda_{1}}{k_{1}} x_{1}^{*}+x_{3}^{*}\right) \\
& +\mu\left(\frac{\lambda_{2}}{k_{2}} x_{2}^{*} x_{3}^{*}+\alpha_{32} \alpha_{23} x_{2}^{*} x_{3}^{*}+\frac{\lambda_{1} \lambda_{2}}{k_{1} k_{2}} x_{1}^{*} x_{2}^{*}+\frac{\lambda_{1}}{k_{1}} x_{1}^{*} x_{3}^{*}+\alpha_{31} \alpha_{13} x_{1}^{*} x_{3}^{*}-\alpha_{12} \alpha_{21} x_{1}^{*} x_{2}^{*}\right) \\
& +\left(\frac{\lambda_{1} \lambda_{2}}{k_{1} k_{2}}+\frac{\lambda_{1}}{k_{1}} \alpha_{23} \alpha_{32}-\alpha_{12} \alpha_{21}-\alpha_{12} \alpha_{31} \alpha_{23}-\alpha_{13} \alpha_{21} \alpha_{32}+\frac{\lambda_{2}}{k_{2}} \alpha_{13} \alpha_{31}\right) x_{1}^{*} x_{2}^{*} x_{3}^{*}=0
\end{aligned}
$$

or $b_{3} \mu^{3}+b_{2} \mu^{2}+b_{1} \mu+b_{0}=0$, where $b_{3}=1, b_{2}=\lambda_{2} x_{2}^{*} / k_{2}+\lambda_{1} x_{1}^{*} / k_{1}+x_{3}^{*}$,

$$
\begin{aligned}
b_{1}= & \left(\lambda_{2} / k_{2}\right) x_{2}^{*} x_{3}^{*}+\alpha_{32} \alpha_{23} x_{2}^{*} x_{3}^{*}+\left(\lambda_{1} \lambda_{2} / k_{1} k_{2}\right) x_{1}^{*} x_{2}^{*}+\left(\lambda_{1} / k_{1}\right) x_{1}^{*} x_{3}^{*} \\
& +\alpha_{31} \alpha_{13} x_{1}^{*} x_{3}^{*}-\alpha_{12} \alpha_{21} x_{1}^{*} x_{2}^{*}, \\
b_{0}= & \left(\left(\lambda_{1} \lambda_{2} / k_{1} k_{2}\right)+\left(\lambda_{1} / k_{1}\right) \alpha_{23} \alpha_{32}-\alpha_{12} \alpha_{21}-\alpha_{12} \alpha_{31} \alpha_{23}-\alpha_{13} \alpha_{21} \alpha_{32}\right. \\
& \left.+\left(\lambda_{2} / k_{2}\right) \alpha_{13} \alpha_{31}\right) x_{1}^{*} x_{2}^{*} x_{3}^{*} .
\end{aligned}
$$

\section{References}

[1] K. S. Chaudhuri, "A bioeconomic model of harvesting a multispecies fishery", Ecol. Model. 32 (1986) 267-279.

[2] K. S. Chaudhuri, "Dynamic optimization of combined harvesting of a two species fishery", Ecol. Model. 41 (1988) 17-25.

[3] K. S. Chaudhuri and T. Johnson, "Bioeconomic dynamics of a fishery modelled as an S-system", Math. Biosci. 99 (1990) 231-249.

[4] K. S. Chaudhuri and S. Saha Ray, "On the combined harvesting of a prey predator system", J. Biol. Syst. 4(1996) 376-389. 
[5] C. W. Clark, Mathematical bioeconomics: the optimal management of renewable resources (Wiley, New York, 1976).

[6] C. W. Clark, Bioeconomic modelling and fisheries management (Wiley, New York, 1985).

[7] M. Mesterton-Gibbons, "On the optimal policy for the combined harvesting of predator and prey", Nat. Resour. Model. 3 (1988) 63-90.

[8] J. D. Murray, Mathematical Biology (Springer, Berlin, 1993).

[9] L. S. Pontryagin, V. G. Boltyonskü, R. V. Gamkrelidre and E. F. Mishchenko, The mathematical theory of optimal processes (Wiley, New York, 1962).

[10] D. L. Ragozin and G. Brown, "Harvest policies and non-market valuation in a predator-prey system", J. Envirn. Econ. Manag. 12 (1985) 155-168. 